\title{
Os benefícios do teletrabalho durante a pandemia covid-19 a um paciente com lesão da medula espinhal parcial por trauma de mergulho em águas rasas: um estudo de caso
}

\author{
The benefits of telework durind the COVID-19 pandemic to a patient with partial spinal \\ cord injury from shallow diving trauma: a case study
}

Los beneficios del teletrabajo durante la pandemia de COVID-19 para un paciente con lesión parcial de la médula espinal por trauma de buceo superficial: un estudio de caso

Thiago Alves Hungaro ${ }^{*}$, Ana Carolina Zanin Sacoman Kurihara1, Jaqueline Ramos de Aguiar², Juliana Pelegrino Simionato Costa ${ }^{2}$, Júlia Aracely Regonha Polizel ${ }^{1}$, Marcel Frisene ${ }^{2}$, Matheus Guimarães Matos ${ }^{1}$, Nathália Tomaz Viccari², Siumara de Paula Tostes Palhares ${ }^{2}$, Tamires Cristina de Souza Cunha².

\section{RESUMO}

Objetivo: Aprofundar o conhecimento sobre o quadro clínico e reabilitação de um usuário com lesão da medula espinhal parcial por trauma de mergulho em águas rasas, analisando a efetividade e os benefícios proporcionados pelo atendimento da equipe em regime de teletrabalho. Detalhamento do caso: Paciente masculino, 38 anos, diagnóstico de traumatismo raquimedular parcial, no nível de C6, há 6 anos, por mergulho em águas rasas. O acompanhamento do paciente iniciou com atendimento no Centro Especializado de Reabilitação em 9 de agosto de 2019, avaliado por equipe multiprofissional e sendo submetido à aplicação da Classificação Internacional de Funcionalidade, Incapacidade e Saúde (CIF). Foi realizada coleta de dados do prontuário, para comparação do quadro cinético funcional anterior e atual. $O$ estudo foi aprovado por Comitê de Ética em Pesquisa, sob o número da CAAE 39677620.0.0000.5384. Considerações finais: Os resultados obtidos neste relato demonstram a importância do teletrabalho durante a pandemia e conclui-se que o teletrabalho realizado pela equipe multiprofissional do Centro Especializado de Reabilitação, foi efetivo para este usuário, permitindo a estruturação e fortalecimento das políticas públicas existentes, assim como a criação de novos protocolos priorizando os seguimentos das atividades multiprofissionais e a evolução clínica do paciente.

Palavras-chave: Teletrabalho, Lesões do pescoço, Reabilitação.

\begin{abstract}
Objective: Deepen the knowledge about the clinical picture and rehabilitation of a user with partial spinal cord injury due to trauma from diving in shallow water, analyzing the effectiveness and benefits provided by the service of the team in telework regime. Case details: Male patient, 38 years old, diagnosed with partial spinal cord trauma, at the level of 66 for 6 years, due to diving in shallow water. Patient follow-up has began with care at the Specialized Reabilitation Center on August 9, 2019, evaluated by a multidisciplinary team and being submitted to the application applied to the International Classification of Functioning, Disability and Health (ICF). Data were collected from the medical records to compare the previous and current functional kinetics. The study was approved by the Research Ethics Committee, under the CAAE number 39677620.0.0000.5384. Final considerations: The results obtained in this report demonstrate the importance of telework during the
\end{abstract}

${ }^{1}$ Centro Universitário Municipal de Franca (UniFACEF), Franca - SP. *E-mail: thungaro@hotmail.com

${ }^{2}$ Centro Especializado de Reabilitação II de Morro Agudo (CER II Morro Agudo), Morro Agudo - SP. 
pandemic and it can be concluded that telework carried out by the Specialized Reabilitation Center multidisciplinary team was effective for this user, allowing the structuring and strengthening of existing public policies, as well as the creation of new protocols prioritizing the segments of multidisciplinary activities and the patient's clinical evolution.

Keywords: Teleworking, Neck injuries, Rehabilitation.

\section{RESUMEN}

Objetivo: Profundizar en el conocimiento sobre el cuadro clínico y rehabilitación de un usuario con lesión de la médula espinal parcial por traumatismo por bucear en aguas poco profundas, analizando la efectividad y los beneficios que aporta para el servicio del equipo en régimen de teletrabajo. Detalles del caso: Paciente de sexo masculino, 38 años, diagnosticado de traumatismo medular parcial, a nivel de C6 desde hace 6 años, por buceo en aguas poco profundas. El seguimiento de los pacientes se inició con la atención en el Centro Especializado de Rehabilitación el 9 de agosto de 2019, evaluados por un equipo multidisciplinario y sometida a la aplicación de la Clasificación Internacional de Funcionamiento, Discapacidad y Salud Se recogieron datos de las historias clínicas para comparar la cinética funcional anterior y actual. El estudio fue aprobado por el Comité de Ética en Investigación, bajo el número CAAE 39677620.0.0000.5384. Consideraciones finales: Los resultados obtenidos en este informe demuestran la importancia del teletrabajo durante la pandemia y se puede concluir que el teletrabajo realizado por el equipo multidisciplinario del Centro Especializado de Rehabilitación fue efectivo para este usuario, permitiendo la estructuración y fortalecimiento de las políticas públicas existentes, así como la creación de nuevos protocolos priorizando los segmentos de actividades multidisciplinares y la evolución clínica del paciente.

Palabras clave: Teletrabajo, Traumatismos del cuello, Rehabilitación.

\section{INTRODUÇÃO}

De acordo com a Organização Mundial da Saúde (OMS), pandemia é uma propagação mundial de uma doença desconhecida com disseminação comunitária, acometendo diferentes continentes, o que levou à recomendação de inúmeras medidas restritivas ao redor do globo (RIBEIRO MTA e CABRAL CHPL, 2020). Os primeiros casos da infecção causada pelo novo coronavírus iniciaram-se no final de 2019, na cidade de Wuhan, na China, e em meados de janeiro de 2020, a OMS considerou-os uma emergência pública internacional, e em março do mesmo ano foi declarado uma pandemia (GAURIAU R, 2020).

A pandemia afetou a rotina das pessoas devido a sua velocidade de contaminação, trazendo inúmeras dificuldades a todos, ao redor do mundo, resultando em um impressionante número de mortes e graves problemas econômicos e sociais (SOUZA DO, 2020).

Esperava-se que o ano de 2020 fosse promissor com o desenvolvimento de várias tecnologias para a área de saúde, no entanto, a crise instaurada em decorrência da Covid-19, gerou instabilidade causando um estado de calamidade pública. Os serviços de saúde, a mobilidade social, a gestão, o planejamento e as operações do Estado e das organizações foram afetados de maneira significativa, desencadeando grandes e graves impactos à população mundial (TING DSW, et al., 2020; CASTRO BLG, et al., 2020).

Mediante o cenário atual, o teletrabalho tem se tornado uma alternativa de trabalho à distância (SANTOS FHS, et al., 2018). Ele está associado ao trabalho realizado remotamente, pela equipe de saúde através de Tecnologias de Informação e Comunicação (TICs). O termo teletrabalho (telecommuting ou telework, em inglês) surgiu na década de 1970, através do trabalho remoto influenciado pela crise energética (petrolífera) (ROCHA CTM e AMADO FS, 2018).

Como estratégia para a não disseminação e enfrentamento da Covid-19, em caráter excepcional e temporário, no âmbito do Sistema Único de Saúde (SUS), houve a necessidade de implementar ações em teletrabalho, para garantir o atendimento com integridade, segurança e sigilo das informações (MINISTÉRIO DA SAÚDE, 2020). 
O Centro Especializado em Reabilitação (CER) é componente da Rede de Atenção Especializada da Pessoa com Deficiência dos municípios, com o objetivo de melhorar a qualidade de vida e a promoção da independência e autonomia dos usuários atendidos, atuando na habilitação e reabilitação da pessoa com deficiência intelectual e física, sendo que este atendimento especializado é executado por equipe multiprofissional composta por fisioterapeutas, terapeutas ocupacionais, fonoaudiólogas, psicólogas, psicopedagoga, nutricionista, equipe de enfermagem, assistente social, ortopedista, psiquiatra adulto e infantil, neurologista e neuropediatra (SECRETARIA DE ESTADO DA SAÚDE DE SÃO PAULO, 2020).

A equipe trabalha em uma perspectiva interdisciplinar e multiprofissional, considerando a individualidade e o impacto da deficiência sobre a funcionalidade do indivíduo, através de avaliação e aplicação da Classificação Internacional de Funcionalidade, Incapacidade e Saúde (CIF), que foi publicada pela OMS em 2001, e é um parâmetro utilizado para classificação da funcionalidade e da incapacidade que integra o modelo biomédico e social (SECRETARIA DE ESTADO DA SAÚDE DE SÃO PAULO, 2020).

O principal objetivo da CIF é possibilitar uma comunicação entre os diversos setores de forma igualitária e universal, através de um modelo teórico de funcionalidade e incapacidade, que permita que a equipe multiprofissional avalie e quantifique o progresso do paciente (BARRETO MCA, et al., 2021). Segundo o Plano de Ação da Rede de Cuidados à Pessoa com Deficiência do Estado de São Paulo, a participação da família é considerada de grande importância na atenção e no cuidado da pessoa com deficiência, além do atendimento multiprofissional, em virtude do seu impacto positivo no processo de reabilitação (SECRETARIA DE ESTADO DA SAÚDE DE SÃO PAULO, 2020).

Dentro do público-alvo prioritário atendido pelo Centro Especializado em Reabilitação, tem-se o Traumatismo Raquimedular (TRM) (SECRETARIA DE ESTADO DA SAÚDE DE SÃO PAULO, 2020). O TRM é uma doença heterogênea podendo variar desde um caso decorrente de uma fratura simples sem déficit neurológico, até casos de lesão neurológica grave e lesões sistêmicas graves (ALVES CL, et al., 2018). É considerado TRM qualquer lesão de causa externa na coluna vertebral, podendo incluir a medula ou raízes nervosas, em qualquer segmento vertebral, e ocasionar danos neurológicos, tais como alterações da função motora, autônoma e sensitiva (ROUANET C, et al., 2017).

O traumatismo raquimedular é caracterizado como uma lesão que acomete uma ou mais partes da coluna vertebral, tanto a parte óssea como a medula espinhal. Fazendo parte das principais causas de morbidade e mortalidade mundial, o TRM gera grande custo à saúde pública devido às incapacidades que causa ao indivíduo acometido, além do impacto causado na vida, visto que a maioria das vítimas se encontra na fase produtiva (MOREIRA RB e SOUZA AM, 2017).

No mundo estima-se entre 9 e 50 casos de TRM por milhão de habitantes; no Brasil os dados referentes a incidência de lesão raquimedular são desconhecidos ou subnotificados, mas sabe-se que é mais comum no sexo masculino, na faixa etária entre 21 e 40 anos e dentre as principais causas de TRM encontramos: acidentes automobilísticos, queda de altura, acidente por mergulho em água rasa e ferimentos por arma de fogo (ALVES CL, et al., 2018).

De acordo com a etiologia a lesão medular do traumatismo raquimedular pode ser de origem traumáticas e não traumáticas. A lesão medular traumática ocorre quando há impacto físico externo, pondendo ser decorrente de um acidente automobilistico, quedas, lesão esportiva ou até mesmo violência; já a lesão medular não traumática ocorre quando há uma patologia aguda ou crônica, dentre elas: tumor maligno ou benigno, infecção ou degeneração osteoarticular da coluna vertebral (AHUJA CS, et al., 2017).

A partir da avaliação pode-se classificar a lesão como completa e incompleta, sendo assim possível avaliar o comprometimento neurológico que determinou as possíveis complicações que este usuário poderia apresentar dentro da sua evolução clínica. Na lesão medular completa não há contração anal voluntária, enquanto que as lesões incompletas são fundamentadas a partir de uma avaliação motora e sensorial detalhada (HARVEY LA, 2016). 
As lesões na medula espinhal podem se manifestar de diversas maneiras, desde uma concussão transitória, com contusão, laceração e compressão da substância da medula, podendo o indivíduo recuperarse completamente; até a casos em que se observa uma transecção incompleta ou completa, levando a paralização abaixo do nível da lesão traumática, manifestando-se com alterações fisiológicas, dentre elas, bexiga neurogênica, intestino neurogênico, espasticidade, úlceras por pressão, pneumonias, choque medular, choque neurogênico, trombose venosa profunda, disrreflexia autônoma, alterações psicossociais e infecções. É importante ressaltar que quando há lesões neurológicas pode haver os riscos de complicações (SOUZA EPD, et al., 2013).

O presente estudo teve por objetivo aprofundar o conhecimento sobre o quadro clínico e a reabilitação de um usuário com diagnóstico de lesão raquimedular parcial por trauma de mergulho em águas rasas, analisando a efetividade e os benefícios proporcionados pelo teletrabalho realizado por equipe multiprofissional em um Centro Especializado em Reabilitação, durante o período de pandemia Covid-19.

\section{DETALHAMENTO DO CASO}

O projeto de pesquisa do estudo foi submetido ao Comitê de Ética e Pesquisa (CEP) do Centro Universitário Municipal de Franca (Uni-FACEF), através da Plataforma Brasil, com aprovação em 16 de novembro de 2020, com o CAAE 39677620.0.0000.5384.

Também foi realizada coleta de dados do prontuário, garantindo o total sigilo e ética conforme Termo de Consentimento Livre e Esclarecido (TCLE), aprovado pelo usuário do serviço e sua cônjuge, sendo os dados utilizados para comparação do quadro cinético funcional anterior e atual para verificação dos benefícios e efetividade do teletrabalho.

Trata-se do caso de um paciente masculino, 38 anos, com diagnóstico de traumatismo raquimedular parcial, no nível de $C 6$ há 6 anos, por mergulho em águas rasas. O acompanhamento no Centro Especializado em Reabilitação iniciou-se em 09 de agosto de 2019. O paciente foi avaliado por equipe multiprofissional e submetido à aplicação da Classificação Internacional de Funcionalidade, Incapacidade e Saúde (CIF). Foi diagnosticada tetraplegia espástica com deficiência grave à moderada das funções do corpo, tais como: funções da dor; funções sensitivas; funções do aparelho cardiovascular, funções dos sistemas hematológico e imunológico; funções do aparelho respiratório; funções do aparelho digestivo; funções dos sistemas metabólico e endócrino; funções geniturinárias e reprodutivas; funções neuromusculoesqueléticas e funções relacionadas aos movimentos, limitações e restrições nas atividades e participação como tarefas e demandas gerais; mobilidade; cuidados pessoais; vida doméstica; áreas principais da vida; vida comunitária; social e cívica.

Devido às restrições existentes às práticas de saúde durante o período pandêmico, a equipe multiprofissional propôs ao paciente e cuidadora que fossem enviados semanalmente mensagens e orientações, via aplicativo WhatsApp. A equipe de terapeutas enviava para o usuário e sua esposa, orientações sobre diferentes atividades e tipos de exercícios para fortalecimento da musculatura abdominal adaptada, dissociação de cinturas, exercícios ativos resistidos adaptados por meio de barra para membros superiores (MMSS), exercícios respiratórios para fortalecimento diafragmático, fortalecimento adaptado para tríceps e peitoral, fortalecimento adaptado de bíceps, lateralização e controle de tronco, exercícios proprioceptivos de tronco e MMSS em posição sentado com encosto e sem encosto. A orientação via teleatendimento permitiu que o usuário realizasse esses exercícios todos os dias, em seu domicílio, com auxílio de seu cônjuge e após a finalização das atividades, os mesmos enviavam feedback das ações para os terapeutas.

O paciente foi submetido à aplicação da CIF, sendo que em um primeiro momento obtiveram-se os dados conforme relatado abaixo (Quadro 1). Ressalta-se que a tabela CIF possui uma linguagem unificada e foi desenvolvida pela OMS, permitindo acesso à informação comum e padronizada mundialmente, proporcionando integralidade do acesso e seguimento do tratamento dos pacientes. 
Quadro 1 - Classificação Internacional de Funcionalidade, Incapacidade e Saúde (CIF), realizada em 08 de agosto de 2019.

\begin{tabular}{|c|c|}
\hline s12000 & Deficiência grave da função na estrutura da medula espinhal cervical \\
\hline b2350 & Deficiência grave da função vestibular de posição \\
\hline b2351 & Deficiência grave da função vestibular de equilíbrio \\
\hline b2352 & Deficiência grave da função vestibular de movimento \\
\hline b260 & Deficiência grave da função de proprioceptiva \\
\hline b265 & Deficiência grave da função tátil \\
\hline b2700 & Deficiência grave da função de sensibilidade à temperatura \\
\hline b2701 & Deficiência grave da função de sensibilidade à vibração \\
\hline b2702 & Deficiência grave da função de sensibilidade à pressão \\
\hline b2703 & Deficiência grave da função de sensibilidade a estímulos nocivos \\
\hline b5250 & Deficiência grave da função eliminação de fezes \\
\hline b5253 & Deficiência grave da função de continência fecal \\
\hline b6200 & Deficiência grave da função da micção \\
\hline b6202 & Deficiência grave da função da continência urinária \\
\hline b7101 & Deficiência grave da função da mobilidade de várias articulações \\
\hline b715 & Deficiência grave da função da estabilidade das articulações \\
\hline b7200 & Deficiência moderada da função de mobilidade do ombro \\
\hline b7201 & Deficiência grave da função da mobilidade da pelve \\
\hline b7202 & Deficiência grave da função da mobilidade dos ossos do carpo \\
\hline b7203 & Deficiência grave da função da mobilidade dos ossos do tarso \\
\hline b7208 & $\begin{array}{l}\text { Deficiência moderada da função da mobilidade dos ossos, outras especificadas } \\
\text { (cotovelo e punho) }\end{array}$ \\
\hline b7308 & Deficiência grave da função da força muscular outras especificadas MMSS \\
\hline b730 & Deficiência grave da função da força muscular \\
\hline b740 & Deficiência grave da função da resistência muscular \\
\hline b770 & Deficiência completa da função relacionada com o padrão de marcha \\
\hline d4358 & $\begin{array}{l}\text { Limitação grave da capacidade de realizar a atividade de mover objetos com os } \\
\text { membros inferiores, outras especificadas }\end{array}$ \\
\hline d4602 & $\begin{array}{l}\text { Limitação grave da capacidade de realizar a atividade de deslocar-se fora da sua casa } \\
\text { e de outros edifícios }\end{array}$ \\
\hline d510 & Limitação grave da capacidade de realizar a atividade de lavar-se \\
\hline d540 & Limitação grave da capacidade de realizar a atividade de vestir-se \\
\hline d640 & Limitação grave da capacidade de realizar a atividade de realizar as tarefas domésticas \\
\hline d4358 & $\begin{array}{l}\text { Restrição grave do desempenho da participação de mover objetos com os membros } \\
\text { inferiores, outras especificadas }\end{array}$ \\
\hline d4602 & $\begin{array}{l}\text { Restrição grave do desempenho da participação de deslocar-se fora da sua casa e de } \\
\text { outros edifícios }\end{array}$ \\
\hline d510 & Restrição grave do desempenho da participação de lavar-se \\
\hline d540 & Restrição grave do desempenho da participação de vestir-se \\
\hline d640 & Restrição grave do desempenho da participação de realizar as tarefas domésticas \\
\hline e310 & Família e serviço de saúde funcionando como facilitador. \\
\hline
\end{tabular}

Fonte: Hungaro TA, et al., 2022; fundamentado em OMS. 2001. ICF: International Classification of Functioning, Disability and Health.

O seguimento da evolução clínica do paciente, durante o tratamento, permitiu observar diversas evoluções, que ficaram evidenciadas quando o mesmo foi novamente submetido à CIF (Quadro 2). Nota-se a maior parte das restrições previamente graves, apresentaram melhora significativa, evoluindo para deficiência moderada, e em alguns casos até para leve. 
Quadro 2 - Classificação Internacional de Funcionalidade, Incapacidade e Saúde (CIF), realizada em 10 de setembro 2020.

\begin{tabular}{|c|c|}
\hline s12000 & Deficiência grave da função na estrutura da medula espinhal cervical \\
\hline b2350 & Deficiência moderada da função vestibular de posição \\
\hline b2351 & Deficiência moderada da função vestibular de equilíbrio \\
\hline b2352 & Deficiência moderada da função vestibular de movimento \\
\hline b260 & Deficiência moderada da função de proprioceptiva de MMSS \\
\hline b265 & Deficiência moderada da função tátil MMSS \\
\hline b265 & Deficiência grave da função tátil MMII \\
\hline b2700 & Deficiência moderada da função de sensibilidade à temperatura MMSS \\
\hline b2700 & Ausência da função de sensibilidade à temperatura MMII \\
\hline b2701 & Deficiência moderada da função de sensibilidade a vibração MMSS \\
\hline b2701 & Deficiência grave da função de sensibilidade a vibração MMII \\
\hline b2702 & Deficiência moderada da função de sensibilidade à pressão MMSS \\
\hline b2702 & Deficiência grave da função de sensibilidade à pressão MMII \\
\hline b2703 & Deficiência grave da função de sensibilidade a estímulos nocivos MMII \\
\hline b5250 & Deficiência grave da função eliminação de fezes \\
\hline b5253 & Deficiência grave da função de continência fecal \\
\hline $\mathrm{b} 6200$ & Deficiência grave da função da micção \\
\hline b6202 & Deficiência grave da função da continência urinária \\
\hline b7101 & Deficiência moderada da função da mobilidade de várias articulações MMSS \\
\hline b715 & Deficiência grave da função da estabilidade das articulações MMII \\
\hline b7200 & Deficiência leve da função de mobilidade do ombro \\
\hline b7201 & Deficiência grave da função da mobilidade da pelve \\
\hline b7202 & Deficiência moderada da função da mobilidade dos ossos do carpo \\
\hline b7203 & Deficiência grave da função da mobilidade dos ossos do tarso \\
\hline b7208 & $\begin{array}{l}\text { Deficiência moderada da função da mobilidade dos ossos, outras especificadas } \\
\text { (cotovelo e punho) }\end{array}$ \\
\hline b7308 & Deficiência leve da função da força muscular outras especificadas MMSS \\
\hline b7308 & Deficiência grave da função da força muscular outras especificadas MMII \\
\hline b740 & Deficiência grave da função da resistência muscular MMII \\
\hline b770 & Deficiência completa da função relacionada com o padrão de marcha \\
\hline d4358 & $\begin{array}{l}\text { Limitação grave da capacidade de realizar a atividade de mover objetos com os } \\
\text { membros inferiores, outras especificadas }\end{array}$ \\
\hline d4602 & $\begin{array}{l}\text { Limitação moderada da capacidade de realizar a atividade de deslocar-se fora da sua } \\
\text { casa e de outros edifícios }\end{array}$ \\
\hline d456 & $\begin{array}{l}\text { Limitação moderada na capacidade de deslocar-se utilizando algum tipo de } \\
\text { equipamento }\end{array}$ \\
\hline d510 & Limitação grave da capacidade de realizar a atividade de lavar-se \\
\hline d540 & Limitação grave da capacidade de realizar a atividade de vestir-se \\
\hline $\mathrm{d} 640$ & $\begin{array}{l}\text { Limitação moderada da capacidade de realizar a atividade de realizar as tarefas } \\
\text { domésticas }\end{array}$ \\
\hline d4358 & $\begin{array}{l}\text { Restrição grave do desempenho da participação de mover objetos com os membros } \\
\text { inferiores, outras especificadas }\end{array}$ \\
\hline d4602 & $\begin{array}{l}\text { Restrição moderado do desempenho da participação de deslocar-se fora da sua casa e } \\
\text { de outros edifícios }\end{array}$ \\
\hline d510 & Restrição grave do desempenho da participação de lavar-se \\
\hline d540 & Restrição grave do desempenho da participação de vestir-se \\
\hline d640 & Restrição moderada do desempenho da participação de realizar as tarefas domésticas \\
\hline e310 & Família e serviço de saúde funcionando como facilitador. \\
\hline
\end{tabular}

Fonte: Hungaro TA, et al., 2022; fundamentado em OMS. 2001. ICF: International Classification of Functioning, Disability and Health. 
Com base na análise da CIF e seus resultados foi possível observar melhora significativa na deficiência das funções vestibular de posição e equilíbrio (b2351), também nas funções proprioceptivas de MMSS (b260), nas funções vestibulares da função tátil de MMSS (b2701); vibração (b2701); sensibilidade a temperatura de MMSS (b2700) e sensibilidade a pressão (b2701) onde houve evolução de grave para moderado.

Já nos aspectos limitação da capacidade de realizar as atividades de deslocar-se fora da sua casa e de outros edifícios (d4602) e deslocar-se utilizando algum tipo de equipamento (d456), houve melhora de grave para moderado. Em relação à restrição do desempenho da participação em realizar as tarefas domésticas (d640) e deslocar-se fora de casa e de outros edifícios (d4602) apresentou uma evolução de grave para moderado.

A realização do teleatendimento, mesmo com orientação e supervisão à distância, proporcionou ao paciente e sua esposa a possibilidade de realizar seguimento supervisionado ao tratamento tão fundamental naquele momento de vida, sempre mantendo o monitoramento por profissionais especializados e capacitados para tal, prevalecendo a excelência clínica e a científica, que se mostraram eficazes e resolutivas para o caso deste paciente, justificando portanto a efetividade do teletrabalho através da sua equipe multiprofissional.

Com a flexibilização das restrições em decorrência da evolução da pandemia de Covid-19 no mundo, o paciente retornou aos atendimentos presenciais, estes aconteceram e continuam sendo realizados em visitas semanais de cerca de 40 minutos. Com a retomada dos atendimentos presenciais foi possível observar todas as melhorias previamente citadas, assim como melhorias em controle postural de tronco, aumento de força muscular nos movimentos de flexão, extensão e abdução de ombro, realização do movimento de alcance, melhora da escrita sem adaptações, melhora nas mudanças de decúbito e sentar no leito diminuindo a necessidade de auxílio da esposa, permitindo mais autonomia e independência ao paciente.

\section{DISCUSSÃO}

Com a pandemia, muitos serviços de saúde começaram a utilizar formas de telessaúde para comunicarse com seus pacientes em casa (HOLLANDER JE e CARR BG, 2020; LUKAS H, et al., 2020). As técnicas propostas para reabilitação neste relato, foram enviadas via aplicativo WhatsApp para o usuário e seu cônjuge, para fortalecimento muscular dos membros superiores utilizando exercícios convencionais e utilização do biofeedback motor. Os exercícios de fortalecimento muscular são eficazes para auxiliar na melhora do controle e estabilização do tronco (BERNARDI CMS, et. al, 2020; MARI KLS, et al., 2019). Mesmo sendo orientadas e supervisionadas à distância, as técnicas empregadas mostraram-se eficazes e auxiliaram na evolução positiva do quadro clínico do paciente relatado no caso, justificando a efetividade do trabalho realizado neste serviço através de sua equipe multiprofissional.

Segundo Hollander JE e Carr BG (2020) nenhum protocolo de teleatendimento é criado de um dia para o outro, no entanto a possibilidade de realizar seguimento 24 horas por dia, facilitou tomada de muitas decisões de maneira objetiva otimizando o acesso, seguimento com segurança para o paciente a equipe assistente. Assim como Santos FHS, et al. (2020) evidenciou que o teletrabalho foi uma alternativa que possibilitou dar continuidade aos trabalhos que foram momentaneamente suspensos devido às restrições sanitárias.

Pappot N, et al. (2020) evidenciou a demanda urgente de expansão dos serviços de teletrabalho, visando otimizar o acesso e agilizar a resolução de problemas graves, direcionando para atendimento presencial após primeira avaliação virtual, assim como manter as orientações e supervisão dos quadros menos graves. Encoranjando os serviços à associações com empresas de tecnologias de teletrabalho, elevando a qualidade dos serviços prestados.

Em seu estudo Temesgen ZM, et al. (2020), reforçou a necessidade de melhorias quando comparado ao atendimento presencial, devido à grande erosão que pode acontecer no vínculo da equipe com o paciente, que pode trazer prejuízos inclusive da percepção de qualidade e valor do mesmo. No entanto, contemplou e enalteceu o importante resultado atingido com o atendimento virtual durante à pandemia, sendo que as organizações de saúde deveriam desenvolver metodologias de aprimoramento do teletrabalho para prover atendimento domiciliar de qualidade e à custos reduzidos. 
Segundo Souza DO (2020), as alterações das funções corporais não estão limitadas apenas à realização de tarefas, mas sofrem também influência de fatores ambientais e pessoais, podendo atuar como barreiras, limitando o desempenho de atividade e a participação social. As intervenções da equipe de reabilitação podem permitir ao indivíduo conviver com a sua incapacidade e melhorar sua qualidade de vida. Portanto, o processo de reabilitação é muito importante para as pessoas vítimas de TRM, e a sua interrupção traz grandes prejuízos à evolução do quadro. A iniciativa do teletrabalho possibilitou a continuidade do tratamento do paciente citado no caso e uma melhora importante da sua funcionalidade.

O teletrabalho realizado por equipe multiprofissional em um Centro Especializado em Reabilitação, foi efetivo para este caso, pois, foram evidenciadas importantes melhorias em diversas funções avaliadas, dentre elas: funções vestibulares, controle de tronco estático e dinâmico, amplitude de movimento ativo de membros superiores, trocas posturais e coordenação motora grossa e fina. A evolução clínica proporcionou melhora da qualidade de vida do usuário, permitindo que apesar de todas as dificuldades inerentes ao lockdown e restrições do período pandêmico, o mesmo tivesse acesso ampliado ao cuidado e seguimento de sua patologia, através de novas tecnologias e instrumentos terapêuticos. O teletrabalho tornou-se importante em situações adversas, como no caso descrito durante a pandemia de Covid-19, interferindo positivamente no prognóstico dos pacientes, garantindo, portanto, a aplicabilidade dos princípios e proporcionando maior efetividade do trabalho proporcionado aos pacientes do SUS.

\section{REFERÊNCIAS}

1. AHUJA CS, et al. Traumatic spinal cord injury. Nature Rewiews Disease Primers, 2017; 3: 1-21.

2. ALVES CL, et al. Trauma raquimedular em paciente de 12 anos com compressão de canal medular sem déficits neurológicos. Revista de Patologia do Tocantins, 2018; 5(1): 41-45.

3. BARRETO MCA, et al. Classificação Internacional de Funcionalidade, Incapacidade e Saúde (CIF) como dicionário unificador de termos. Acta Fisiatr., 2021; 28(3): 207-213.

4. BERNARDI CMS, et al. Intervenção Fisioterapêutica no controle de tronco em um paciente com trauma raquimedular (TRM): relato de caso. Revista Científica da Saúde, 2020; 2(1): 48-56.

5. CASTRO BLG, et al. COVID-19 e organizações: estratégias de enfrentamento para redução de impactos. Rev. Psicol. Organ. Trab., 2020; 20(3):1059-1063.

6. GAURIAU R. Teletrabalho em tempos de COVID-19: Estudo comparado Franco-Brasileiro. Rev. do Trib. Reg. Trab. 10를 Região, 2020; 24(1): 219-230.

7. HARVEY LA. Physiotherapy rehabilitation for people with spinal cord injuries. Journal of Physiotherapy, 2016; 62:4-11.

8. HOLLANDER JE, CARR BG. Virtually Perfect? Telemedicine for COVID-19. N engl j med, 2020; 382(18): 1679-1681.

9. LUKAS H, et al. Emerging Telemedicine Tools for Remote COVID-19 Diagnosis, Monitoring, and Management. ACS Nano, 2020; 14(12): 16180-16193.

10. MARI KLS, et al. Técnicas fisioterapêuticas utilizadas na reabilitação de pacientes com lesão medular- Estudo de Revisão. Connectionline, 2019; 20: 46-56.

11. MINISTÉRIO DA SAÚDE. Secretaria de Atenção Primária à Saúde (SAPS). Manual do Ministério da Saúde / SAPS Protocolo de manejo clínico do Coronavírus (Covid 19) na atenção primária a saúde. Brasília - DF: Brasil, 2020. Disponível em: https://saude.rs.gov.br/upload/arquivos/202004/14140606-4-ms-protocolomanejo-aps-ver07abril.pdf. Acesso em: 24 jan. 2022.

12. MOREIRA RB, SOUZA AM. Significados da deficiência adquirida na vida adulta: implicações do trauma raquimedular. Psicologia em Estudo, 2017; 22(2): 243-251.

13. ORGANIZAÇÃO MUNDIAL DA SAÚDE (OMS). 2001. International Classification of Functioning (ICF), Disability and Health. Suíça. Disponível em: https://apps.who.int/iris/bitstream/handle/10665/42407/9788531407840_por.pdf?sequence=111. Acesso em: 24 jan. 2022.

14. SECRETARIA DO ESTADO DA SAÚDE DE SÃO PAULO. Plano de Ação da Rede de Cuidados à Pessoa com Deficiência do Estado de São Paulo. Coordenadoria de Planejamento de Saúde, Grupo Técnico de Ações Estratégicas III, Grupo Condutor Estadual da RCPD, 2020. Disponível em: https://www.cosemssp.org.br/wpcontent/uploads/2021/04/Plano-Estadual-RCPD-Versao-Final-dia-29.09.pdf. Acesso em: 24 jan. 2022.

15. ROCHA CTM, AMADO FS. O teletrabalho: conceituação e questões para análise. Cad. EBAPE.BR, 2018; 16(1): 152162. 
16. ROUANET C, et al. Traumatic spinal cord injury: current concepts and treatment update. Arq Neuropsiquiatr, 2017; 75(6): 387-393.

17. RIBEIRO MTA, CABRAL CHPL. A dignidade humana frente às medidas sanitárias restritivas da OMS e dos estados em tempos de pandemia. Cadernos Eletrônicos Direito Internacional Sem Fronteiras. 2020; 2(1): 1-22.

18. SANTOS FHS, et al. A importância do contrato de teletrabalho durante a pandemia do (COVID-19). Revista Interciência, 2020; 1(4): 2-7.

19. SOUZA DO. A pandemia de COVID-19 para além das Ciências da Saúde: reflexões sobre sua determinação social. Ciência \& Saúde Coletiva, 2020; 25(1): 2469-2477.

20. SOUSA EPD, et al. Principais complicações do Traumatismo Raquimedular nos pacientes internados na unidade de neurocirurgia do Hospital de Base do Distrito Federal. Com. Ciências Saúde, 2013; 24(4): 321-330.

21. TING DSW, et al. Digital technology and COVID-19. Nature Medicine, 2020; 26(4): 459-461.

22. PAPPOT N, et al. Telemedicine and e-Health Solutions for COVID-19:Patients' Perspective. TELEMEDICINE and eHEALTH, 2020; 26(7): 847-849.

23. TEMESGEN ZM, et al. Health Care After the COVID-19 Pandemic and the Influence of Telemedicine. Mayo Clin Pro, 2020; 95(9S): S66-S68. 